\title{
Criminologie
}

\section{Table ronde sur la peine de mort}

\section{Fernando Acosta}

Volume 20, numéro 2, 1987

La détermination de la sentence

URI : https://id.erudit.org/iderudit/017254ar

DOI : https://doi.org/10.7202/017254ar

Aller au sommaire du numéro

Éditeur(s)

Les Presses de l'Université de Montréal

ISSN

0316-0041 (imprimé)

1492-1367 (numérique)

Découvrir la revue

Citer ce document

Acosta, F. (1987). Table ronde sur la peine de mort. Criminologie, 20(2), 103-113. https://doi.org/10.7202/017254ar d'utilisation que vous pouvez consulter en ligne.

https://apropos.erudit.org/fr/usagers/politique-dutilisation/ 
Le 30 juin dernier, la Chambre des communes rejetait, par 148 voix contre 127, une motion du gouvernement conservateur qui appuyait en principe le rétablissement de la peine de mort au Canada et, du même coup, prévoyait le déclenchement d'un processus pouvant conduire à l'inclusion de cette mesure dans la loi pénale du pays. Dans les jours qui précédèrent ce vote historique, bien peu furent ceux qui risquèrent un quelconque pronostic. On raconte même que, à peine quelques heures avant la délibération, certains députés craignaient la possibilité d'un résultat nul, éventualité qui placerait bien entendu le président de la Chambre dans la dramatique position de trancher la question. Deux jours plus tard, la victoire bien savourée, pour les uns, la défaite mal digérée, pour les autres, la décision des Communes était déjà chose du passé et les médias d'information s'affairaient, comme il fallait s'y attendre, à la découverte/création de nouvelles actualités. Ce que l'on a convenu d'appeler "débat sur la peine de mort" venait-il de connaître son épilogue, et pour de bon cette fois-ci, suite au vote abolitionniste extrêmement serré (130 contre 124) de juillet 1976 ?

Il est au moins deux raisons de croire que non. La première tient sans doute au fait que peu de questions se sont montrées, au cours de l'histoire, aussi récurrentes que celle de savoir si certaines fautes devraient ou non se payer au prix de la vie. La deuxième découle de la constatation qu'au-delà des empoignades dont cette question fut souvent le motif ou prétexte, aussi bien au plan de la morale que sur le terrain glissant du sens commun, elle demeure un sujet d'âpre controverse dans les domaines de réflexion respectifs du droit et de la criminologie.

Deux raisons, suffisantes nous semble-t-il, pour que nous décidions d'inaugurer cette nouvelle section de Criminologie - alors que la fameuse question plonge à nouveau (pour combien de temps?) dans son sommeil hibernal - avec des extraits des interventions de quatre universitaires que nous avons réunis, le 26 mai dernier, pour débattre de quelques-uns des principaux enjeux entourant le rétablissement de la peine capitale. Il s'agit, dans l'ordre de leur apparition dans le texte qui suit, des professeurs Pierre Landreville (criminologie, Université de Montréal), Guy Rocher et Hélène Dumont (droit, Université de Montréal) et Alvaro Pires (criminologie, Université d'Ottawa).

* Professeur au Département de criminologie à l'Université d'Ottawa. 
L'initiative de cette rencontre, qui eut lieu à un moment où les abolitionnistes et nostalgiques de l'échafaud s'affrontaient de plus belle sur la place publique, procédait d'une constatation que nous avons transmise à nos invités sous la forme de la question consigne suivante: est-il possible d'élargir l'actuel débat sur la peine de mort de manière qu'il porte sur des questions autres que celles de nature morale ou alors, comme il arrive si souvent, celles relatives à l'éternelle querelle autour de l'effet dissuasif de cette forme de punition? Il convient également de rappeler que cette tentative d'approfondissement d'un débat qui pêchait souvent par redondance constituait, au moment où elle fut entreprise et de la part de ceux et celles qui participent à l'organisation de cette revue, une manifestation d'appui intransigeant au maintien de l'abolition de la peine capitale au pays. Que les inévitables contraintes d'espace nous obligent aujourd'hui à ne publier qu'une version abrégée des échanges qui eurent lieu, cela ne saura empêcher, nous en sommes convaincus, que chacun(e) de nos lecteurs et lectrices puisse y trouver suffisamment de matière pour en faire un bilan fort intéressant et utile. Utile, sans aucun doute, pour se préparer à une éventualité contre laquelle il paraît assez laborieux de se prémunir, soit que la proverbiale étroitesse d'esprit des «rétabolitionnistes", à nouveau, s'étale impunément au grand jour. Les tenants de la ligne dure se plient mal, et pour cause, aux évidences.

Signalons, en guise de conclusion, que les interventions faites au cours de cette table ronde furent enregistrées en totalité sur bande magnétique. Comme il est de coutume dans de telles circonstances, le texte qui suit constitue une version, adaptée aux exigences de l'écriture, de cet ensemble d'interventions orales.

F. Acosta : Comment interpréteriez-vous les résultats des récents sondages d'opinion publique qui indiquent qu'environ $70 \%$ de la population canadienne est favorable au rétablissement de la peine de mort? En d'autres termes, peut-on conclure de ces résultats que la grande majorité des Canadiens non seulement appuient l'idée du rétablissement de la peine capitale mais souhaitent également qu'elle soit effectivement appliquée au pays?

P. Landreville: Il faut rappeler, avant tout, que l'opinion publique est en général très mal informée sur cette question. Elle $\mathrm{a}$, en effet, des perceptions à ce sujet qui contredisent la réalité au moins sur deux points. Sur la criminalité de violence, d'une part, puisque certains sondages au Canada ont montré que la population croit qu'il y a beaucoup plus de criminalité de violence - et je parle de violence dans le sens traditionnel du terme - qu'il y en a en réalité. De là la peur du crime que l'on connait. D'autre part, on constate qu'elle est également mal informée sur le fonctionnement de l'administration de la justice. On a beau rappeler qu'il 
existe une peine de 25 ans d'incarcération pour les individus condamnés pour meurtre au premier degré et les gens vous diront: «oui, mais ils ne les purgeront pas». Or il est possible qu'en s'appuyant sur ces deux faux postulats - c'est-à-dire qu'il y a beaucoup de violence et que, en plus, le système de libérations conditionnelles libère tout le monde rapidement la population arrive à la conclusion que la seule solution au problème est la peine de mort. Au moment de prendre une décision en cette matière, le législateur devra donc tenir compte du fait que s'il suit aveuglement la soi-disant opinion publique il suit en fait deux faux postulats. C'est là, à mon avis, un premier élément à considérer pour que l'on puisse ensuite prendre les décisions les plus éclairées, aux points de vue éthique et politique, sur cette question.

G. Rocher: Je suis toujours porté à prendre au sérieux les résultats des sondages d'opinion publique. Je crois qu'ils sont réels, malgré tout. En effet, il y a probablement une assez importante majorité de la population qui, lorsqu'on lui pose la question, est d'accord avec le rétablissement de la peine de mort. Cependant, cela ne veut pas dire que, devant chaque cas où le juge peut ou doit imposer cette peine, les gens seront d'accord à ce qu'elle s'applique effectivement. En d'autres mots, la sensibilité que l'on a à l'égard de l'être humain joue, jusqu'à un certain point, en faveur de la peine de mort et contre son application - et cela chez le même individu. Ce qui montre, à mon avis, combien il est difficile de faire des sondages, en particulier sur une question aussi complexe. Car il faut se demander à quoi pense l'interviewé lorsqu'on lui pose une telle question, à quel meurtre se reporte-t-il? Est-ce que c'est au meurtre passionnel ou au meurtre d'un enfant? Est-ce que c'est au meurtre d'un gardien de prison ou d'un policier, autour duquel on a fait beaucoup de publicité, ou est-ce que c'est au meurtre commis par un policier en train d'arrêter un suspect? De quel meurtre s'agit-il puisqu'il y en a une grande variété? Or un sondage d'opinion ne peut pas aller dans le détail de cette variété de meurtres, si bien que devant une question aussi globale je crois que l'interviewé va répondre en pensant aux cas les pires, aux cas limites, et non pas à l'ensemble des cas dont il faut tenir compte. Ce qui pose, par conséquent, le problème de savoir, d'une part, qu'est-ce qu'un cas limite de meurtre et, de l'autre, si de tels cas doivent être punis de la peine capitale. C'est le problème qui est posé en ce moment par le procès de Klaus Barbie, en France, à la conscience non seulement française mais internationale: est-ce que les crimes de guerre ou contre l'humanité méritent la peine de mort?

H. Dumont : J'accorde également beaucoup de sérieux aux sondages d'opinion publique. J'y vois l'expression d'une vérité, pas de toute la 
vérité, bien entendu, mais au moins de celle des sentiments de ceux qui répondent aux questions. C'est une réponse dans l'ordre des sentiments et des instincts et, probablement parce que la question est générale, les gens ont, en fait, à l'esprit des cas qui provoquent dans leur imaginaire les sentiments les plus forts, comme la peur, l'horreur. Face à quelque chose qui est perçu comme étant si importante, ils répondent par une solution définitive, par l'éradication du problème: la peine de mort c'est l'éradication du problème. Or, je demeure cependant persuadée que ces gens seraient les tenants d'une autre vérité s'ils pouvaient s'insérer dans la trame du procès pénal, même dans les cas les plus horribles. On a pu souvent constater ce type de contradiction dans des cas, par exemple, de procès pour avortement où les membres du jury — qui étaient, de façon générale, contre cette pratique - ont acquitté les accusés. Or, je ne pense pas que ces personnes soient nécessairement incohérentes, j'estime plutôt qu'à travers cette expérience elles sont confrontées à deux phénomènes en fait différents, à deux niveaux de vérité. Dans ce même ordre d'idées, je crois alors que l'attitude répressive que l'on constate chez les Canadiens est en réalité beaucoup moins grande que ce que nous laissent croire les réponses aux sondages. Quant à la question des crimes contre l'humanité, soulevée par Guy Rocher à la fin de son intervention, je dois dire que je suis contre la peine de mort même dans un cas comme celui de Klaus Barbie, cas qui reste pour moi absolument horrible et intolérable. En fait, dire non à la peine de mort, dans une situation comme celle-là, c'est dire — du moins symboliquement - que je suis contre la violence y compris la violence de Barbie, que je me refuse d'accepter une telle solution dans le contexte d'une démocratie. Et je pense qu'il y a une éducation de société à faire en optant pour un autre moyen que la peine de mort, même à l'encontre d'actes de cette violence commis contre l'humanité. Car ce ne sont pas les victimes de Barbie qui le châtient, c'est la société qui le fait, ce qui me fait dire qu'il y a un geste de civilisation, qu'il y a une valeur symbolique à préserver en n'optant pas, face à une telle situation, pour la peine capitale.

F. Acosta: Au cours des derniers mois, le gouvernement n'a pas cessé d'invoquer l'existence du fameux «engagement électoral» comme justification au déclenchement du débat sur la peine de mort. Voyez-vous d'autres raisons que celle-là qui pourraient expliquer le retour, onze ans plus tard, de cette question à la scène politique?

A. Pires: Il ne me semble pas, en tout cas, que ce retour puisse être interprété comme étant le résultat d'une revendication populaire précise. Je me demande, en d'autres termes, si cet appui au rétablissement de la peine de mort, dont les sondages font état, exprime vraiment une volonté, une revendication pressante de la population. Je me rappelle, par 
exemple, de certaines enquêtes d'opinion faites par le Solliciteur général du Canada où la criminalité et la peine de mort ne figuraient même pas parmi les principaux problèmes qui préoccupaient les répondants. Et voilà que du jour au lendemain le débat sur la peine capitale émerge fort d'un appui aussi massif. Je m'interroge alors sur la force véritable de cette revendication. A-t-elle vraiment du poids ou est-ce qu'il s'agit plutôt de quelque chose qui a été construit, du moins partiellement, à partir de certaines demandes qui ne constituent finalement qu'un souci, parmi bien d'autres, dans la vie quotidienne en société?

H. Dumont : Le programme du parti conservateur contient effectivement des dispositions très précises en faveur de la peine de mort. Et même si le Premier ministre a déjà laissé entendre que, personnellement, il s'oppose à cette sanction, le parti politique qu'il dirige a fait état de demandes spécifiques dans le sens de rétablir cette peine. D'autre part, avant même que l'on ouvre le débat proprement dit, on a assisté à des tentatives venant des députés, de déclencher le processus législatif d'examen de la question. Or, je crois que ces demandes pressantes de certains députés, fondées sur leurs convictions personnelles, inscrites dans un parti politique dont le programme envisage le rétablissement de la peine de mort, ont créé suffisamment de pression pour que le gouvernement se décide à ouvrir le débat. Cela me surprendrait, par contre, d'apprendre que cette initiative vient de l'intérieur de la machine gouvernementale, de ce que j'appellerais les ministères concemés.

G. Rocher : Comment se fait-il qu'un parti politique a inscrit un tel engagement dans son programme? - c'est là la question. Je ne sais pas exactement d'où cela vient, mais ce qui m'apparaît cependant sûr c'est que le programme du parti conservateur a été présenté à l'opinion publique comme étant la proposition d'un parti qui voulait remettre de l'ordre dans une société où l'on considérait qu'il y avait eu un certain désordre jusque-là, où l'on avait trop contesté l'ordre social. Avec une telle perspective, je crois que le parti conservateur profitait d'un moment où la contestation de l'ordre social allait en diminuant, car on n'avait pas, à la fin des années 70, la contestation qu'on a eue dans les années 60-70. Or, la question de la peine de mort s'inscrit bien sûr dans une problématique plus large et, dans ce sens, je crois que, en saisissant chaque occasion de meurtre de policier pour relancer cette question devant l'opinion publique, le lobby des chefs de police a été un moteur important dans le processus de déclenchement de ce débat. Ils ont sans doute réussi à avoir assez d'écoute dans les médias pour convaincre un parti politique, qui cherche à prendre le pouvoir, qu'il y avait là matière d'intérêt pour une opinion publique soucieuse de rétablir l'ordre social. 
P. Landreville: Il ne faudrait sans doute pas perdre de vue qu'il y a une fragmentation importante de l'opinion publique au Canada. Il faut considérer, d'une part, qu'il y a certaines provinces où l'on voulait, beaucoup plus que dans d'autres, rétablir les valeurs morales, et de l'autre, que même l'association nationale des chefs de police s'est montrée de plus en plus prudente dans la revendication du rétablissement de la peine de mort. Je dirais alors que ce sont probablement certains noyaux durs, certains députés qui ont des électorats beaucoup plus conservateurs que l'on peut s'imaginer même au Québec, qui ont poussé cette cause. C'est en effet très symbolique, dans un mouvement d'assainissement de la société, que cela passe entre autres par le rétablissement de la peine de mort - ce qui me fait penser qu'il y a eu effectivement une croisade morale pour la peine de mort, à l'intérieur du parti conservateur, menée par un certain nombre de députés. Je vois d'ailleurs dans l'actuel débat qui se déroule à la Chambre une tentative, de la part de quelques députés d'arrière-banc, de montrer que le parti conservateur ne respectait pas une de ces promesses électorales, n'était pas à vrai dire assez conservateur.

G. Rocher: C'est vrai, comme le dit Pierre Landreville, qu'il y a toujours eu un certain nombre de députés qui croyaient à cette cause. Mais pour l'ensemble du parti conservateur, je crois que, au moment où cette proposition a été acceptée par leur congrès, c'était plutôt de l'opportunisme électoral, cela ne traduisait pas une profonde conviction de la majorité des membres du parti. Par contre, la décision de rouvrir le débat m'apparaît être une erreur politique car je ne vois pas quel intérêt électoral on puisse en tirer en ce moment. Mais tout compte fait, je me dis bien qu'un débat comme celui-là fait mûrir l'opinion publique dans une démocratie, même si je considère qu'on n'aurait jamais dû le déclencher. De fait, si jamais on approuve ce projet on posera un tel problème à l'opinion publique qu'il va falloir qu'on se penche à nouveau sur cette question, vu qu'il me paraît impensable que l'on rétablisse d'une manière définitive la peine de mort au Canada. Elle est, comme on l'a déjà dit, trop difficile à gérer. Les hommes politiques voudront eux-mêmes évacuer cette responsabilité et beaucoup parmi ceux qui aujourd'hui l'approuvent ne tarderont probablement pas à changer d'avis. Sans vouloir la politique du pire, je me dis que c'est peut-être souhaitable que l'on rétablisse la peine de mort pour faire mûrir une opinion publique qui autrement ne mûrirait peut-être pas sur une telle question. La confronter à son propre choix, dit en d'autres mots. Parce que si c'est vrai que 70\% de la population veut la peine capitale, alors ce ne serait peut-être pas un mal que les Canadiens soient confrontés avec les conséquences de ce qu'ils demandent. C'est quand même une manière de concevoir la démocratie... ou le mûrissement de la démocratie. 
H. Dumont: Je ne suis pas tout à fait d'accord avec l'idée d'une expérience de peine de mort au Canada dans le but d'éduquer l'opinion publique. D'une façon extrêmement cynique, je dirais plutôt que si $\mathrm{j}$ 'avais à rédiger une législation en cette matière j'obligerais les responsables de la décision à assister à l'exécution. J'obligerais, par exemple, le juge qui a prononcé une sentence de peine de mort à être présent lors de l'exécution, bref à porter toutes les conséquences de la décision qu'il a rendue. Car je trouve que l'un des dangers qui entourent le rétablissement de la peine capitale c'est qu'on n'est pas vraiment conscient des difficultés concernant sa gestion. On a établi une distance entre ceux qui décident qu'elle doit s'appliquer dans un cas concret et ceux qui l'appliquent effectivement, et c'est en quelque sorte ce qui la rend possible. Tout comme l'adoucissement que l'on constate, au plan des méthodes d'exécution, cette distanciation physique entre l'auteur et l'exécutant de la décision m'apparait comme une stratégie de plus pour qu'elle soit remise en opération.

P. Landreville: Je suis d'accord avec l'idée que l'on dilue les responsabilités à l'intérieur de ce processus avec, cependant, une réserve: le cabinet du Premier ministre, lui, il devra se prononcer d'une façon bien précise sur des cas concrets de condamnation à mort. Ce qui me fait dire, par ailleurs, que ce pouvoir de commutation risque de provoquer des dissensions importantes au sein du cabinet et de placer un certain nombre de ministres dans des positions intolérables. Si l'on regarde, d'ailleurs, les statistiques de 1867 à 1962, on constate que l'on a commué une peine de mort sur deux et, au cours des quinze dernières années de cette période, on retrouve un seul cas d'exécution contre trois commutations. On va donc de plus en plus vers des peines de mort commuées. Et si on n'a pas eu d'exécution au Canada entre 1962 et 1976 c'est, entre autres, parce que ceux qui ont le pouvoir de confirmer en dernière instance une sentence de mort se montrent incapables de prendre une telle décision ou ne veulent pas la prendre.

A. Pires: J'aimerais attirer l'attention sur un aspect de la question qui relève cette fois-ci des droits de l'homme. En effet, on peut dire que si nous avons un certain espoir par rapport à ce qui vient d'être dit autour de cette table, c'est-à-dire qu'une politique élargie de confrontation physique peut faire échec à l'application de la peine de mort, ce n'est sûrement pas parce qu'une telle stratégie a toujours fonctionné, puisque ce n'est pas le cas. Que l'on se souvienne seulement que dans le passé les supplices se faisaient en public et en présence d'un certain nombre d'autorités. Si nous avons l'espoir que cela puisse aujourd'hui produire des effets positifs, c'est probablement parce que nous faisons un vote de 
confiance dans une certaine évolution de la conscience éthique, de la sensibilité populaire. C'est sûr que la proximité physique a un impact important sur le geste à poser, et la fameuse expérience de Milgram le démontre largement. Mais il ne faut pas oublier la part des rationalisations possibles, des représentations que l'on peut se faire dans une telle situation, de sorte que, tout compte fait, bon nombre de personnes seraient quand même capables d'aller jusqu'au bout. Comme Hélène Dumont, je pense également que s'il fallait que l'on rétablisse la peine de mort il serait sans doute mieux que cela se fasse de manière que ceux qui prennent la décision se voient obligés de l'assumer, de la vivre entièrement, et non pas de la déléguer successivement, de la morceler à un point où on ne voit plus qui en est le responsable. Mais je ne crois pas que ce soit là la seule façon de trancher ce débat.

F. Acosta: Un éventuel rétablissement de la peine de mort aurait-il un effet quelconque sur l'échelle des peines effectivement appliquées par les tribunaux, en particulier en matière de crimes contre la personne?

P. Landreville: Si la peine de mort est rétablie au Canada il y a effectivement des effets dangereux à craindre, y compris un effet important sur l'échelle des peines - et cela, même si les sentences de condamnation à mort ne seront pas mises en application, comme plusieurs le croient de plus en plus. Je pense, en effet, que l'échelle des peines garde une relative proportionnalité interne, de nature plutôt symbolique, et que si on ajoute par le haut une peine encore plus sévère que celle de 25 ans d'emprisonnement, on risque de voir apparaître un nouveau point de référence. On pourrait alors s'attendre à un effet d'entrainement vers le haut, dans l'échelle des peines appliquées, qui ne serait probablement pas négligeable. Et même si le gouvernement devra, comme je le crois, rouvrir cette question dans un avenir rapproché, d'ici là on étirera vraisemblablement l'échelle des peines. Un autre effet à craindre d'un éventuel rétablissement de la peine capitale serait celui de banaliser la peine d'incarcération de 25 ans qui deviendrait, dans des telles circonstances, le deuxième châtiment, alors que la peine de mort ne sera de toute évidence pas appliquée. Bref, toute augmentation d'une peine en particulier $\mathrm{a}$, à mon avis, un effet sur les autres.

A. Pires: Qu'il y aura un effet de banalisation de la peine de 25 ans, comme le prédit Pierre Landreville, cela m'apparaît également sûr. En ce qui concerne cependant, une augmentation effective dans l'échelle des peines, je dois dire que je n'y crois pas beaucoup. Je pense plutôt que l'effet le plus pervers que le rétablissement de la peine de mort pourrait avoir, ce serait celui de faire obstacle au principe de modération revendiqué, depuis longtemps, dans la philosophie pénale canadienne et, avec 
beaucoup d'insistance, par le rapport Ouimet et par la Commission de réforme du droit du Canada. En d'autres termes, j'estime qu'un tel rétablissement empêcherait en fait une quelconque réduction dans une échelle de peines qui est déjà très élevée. Dans des pays qui utilisent des échelles de peines moins sévères que la nôtre, comme la Hollande, par exemple, lorsqu'ils ont haussé les sentences pour les délits relatifs aux drogues - dans le cadre d'une politique internationale de répression de ces infractions - ils ont effectivement gonflé les sentences d'emprisonnement pour les autres délits. Mais dans le cas du meurtre, qui entre dans une catégorie à part et, en plus, dans un contexte où les peines sont déjà trop sévères, je ne pense pas que l'ajout représenté par la peine de mort pourrait avoir un tel effet. La conséquence que je crains d'un éventuel rétablissement de la peine capitale est bien plus celle de faire échec à une politique effective de modération des peines.

H. Dumont : Je me demande jusqu'à quel point, dans l'ordre des idées scientifiques, on n'a pas en quelque sorte contribué à ce que l'on envisage le rétablissement de la peine de mort. En effet, lorsqu'on examine l'état des questions sur les fonctions du châtiment - traditionnellement conçues comme étant l'intimidation, la dissuasion, la rétribution, la réhabilitation - on se rend compte que les données de la science ont fait un peu la démonstration du caractère extrêmement problématique de ces fonctions. Relativité, d'abord, de la fonction dissuasive. Échec de la fonction de réhabilitation des peines. Or, qu'est-ce que l'on a bâti dans la réforme moderne? Je dirais, essentiellement des modèles de just deserts policy, c'est-à-dire de juste rétribution. Et je me demande, en effet, dans quelle mesure cette politique ne contribue pas, au plan des idées, à ce que l'on envisage la possibilité de la peine de mort, car elle nous ramène à l'idée que tout ce que l'on peut faire correctement en ce moment c'est de punir de façon égalitaire des personnes semblables. Dans une telle perspective, il est alors inévitable que, face à des actions qui apparaissent plus crapuleuses, la recherche de la juste punition nous amène à considérer que pour les fautes graves il faut une sanction plus sévère. Ne croyant plus à la certitude, ni de la dissuasion ni de la réhabilitation, on se résigne à faire seulement ce que l'on est en mesure de faire, c'est-à-dire traiter des cas semblables de façon semblable, chercher une gradation mathématique, d'abord, dans l'ordre de gravité des actes et, ensuite, dans l'ordre de gravité des châtiments. Or, cette crise des valeurs dans le domaine de la pénologie entraîne aussi une certaine désespérance: il n'y a plus de croyance dans la valeur des peines. Il devient alors plus tolérable de choisir des solutions ultimes, des solutions qui signalent en quelque sorte un échec, qui indiquent qu'il n'y a plus de stratégie en faveur d'une solution d'espoir. Je me demande donc jusqu'à quel point cette crise dans 
l'ordre des valeurs, en matière de châtiment, ne rend-elle pas possible, elle aussi, le rétablissement de la peine de mort.

A. Pires: Je suis d'accord avec ce que soulève Hélène Dumont avec cependant une réserve, que je formule d'ailleurs sur un mode plutôt interrogatif. On a effectivement évacué de la politique des châtiments une sorte de souffle humaniste, représenté par l'idée de la réhabilitation, tout en y laissant ce que $\mathrm{j}$ 'appellerais sa mécanique. Cependant, ce qui constitue pour moi un problème, ce n'est pas le fait qu'on ait perdu la foi dans la punition, mais plutôt qu'on soit resté avec la punition en ayant en même temps perdu l'espoir au sujet de ce que l'on pensait que, par son biais, on pouvait atteindre. Si nous avions été capables de sortir complètement du cadre de la punition, si nous avions essayé de penser le pénal non pas comme quelque chose de naturalisé et autonome - autonome par rapport au reste du droit - on serait peut-être plus en mesure aujourd'hui de proposer des options nouvelles. Je me demande, d'autre part, si cette désespérance est aussi répandue au niveau du grand public qu'elle l'est chez ceux et celles, comme nous, qui se retrouvent quand même relativement proches des instances de décision. Sans trop m'avancer sur ce terrain pour l'instant, $\mathrm{j}$ ' ai quand même l'impression que la croyance dans la réhabilitation demeure encore très forte dans l'opinion publique.

G. Rocher: Je remarque seulement qu'on a vécu cette même expérience en éducation. Éduquer c'était assurer le succès de la personne et, par conséquent, le succès de la société. Il y avait comme un effet mécanique qui en découlait. Tout comme dans le domaine de l'éducation, je crois qu'il y a eu une sorte de pensée mécanique qui a attribué à la réhabilitation un succès nécessaire, automatique. On constate alors un certain désenchantement dans les pratiques des sciences humaines parce que cet automatisme ne se révèle pas bien souvent. Le retour à un certain humanisme devient ainsi difficile, car l'être humain n'est pas la mécanique que peut-être les sciences humaines ont un peu trop laissé entendre qu'il était.

F. Acosta: Y a-t-il des liens à faire entre ces revendications - quels qu'en soient le poids et la signification - pour le rétablissement de la peine capitale et d'autres débats contemporains qui ont mobilisé au moins certains secteurs de ce que l'on appelle l'opinion publique?

G. Rocher: Le débat sur la peine de mort se situe bien sûr dans le contexte d'autres débats, que j'appellerais politico-juridiques, qui nous ont accompagnés depuis une vingtaine d'années: débat sur les droits de la personne, sur l'égalité des sexes, sur l'avortement, sur la torture organisée par les États, qui continue d'ailleurs à exister dans un grand nombre de pays. Peut-on y voir des contradictions ou est-ce que tout cela fait 
finalement une certaine unité? Je me demande, par exemple, si le débat sur les droits de la personne, qui au cours des dernières années nous a sensibilisé au problème des victimes d'actes criminels, n'a pas eu un effet pervers sur le débat sur la peine de mort. La question des victimes, me semble-t-il, est aujourd'hui partie intégrante de ce débat. La victime du meurtre - on pourrait sans doute dire - $\mathbf{a}$ en quelque sorte droit à une rétribution posthume égale au tort subi. Bien entendu, la notion d'égalité entre le dommage subi par la victime et la punition infligée au coupable n'est pas nouvelle, mais elle a évolué dans le temps. De nos jours, il me semble que les droits de la personne sont un peu devenus les droits de la victime, ou inversement. La victime, en d'autres termes, est une personne qui a droit à la punition de son agresseur. Je me demande en effet si ce rapport victime-coupable n'est pas présent dans ce débat, bref, dans la sensibilité publique. Et en disant victime, je pense bien entendu à l'enfant, au policier, au gardien de prison. Pas à l'amante tuée par son amant, ni à l'ouvrier de la construction, ni aux travailleurs des mines.

P. Landreville: Et même dans le cas de l'amante tuée par son amant, on constate que les revendications des mouvements des victimes, des mouvements féministes, vont décrier le fait que certains amants qui tuent leurs maîtresses ne sont pas assez punis. Trois ans pour un tel crime, ce n'est pas suffisant. Or, au fond, cela fait partie de ce même mouvement dans lequel on accorde de plus en plus d'attention à la personne, on tolère de moins en moins la violence et, paradoxalement, tout en tolérant moins la violence on veut défendre la vie par des moyens violents. La demande de peines de plus en plus sévères va ainsi en augmentant et, à la limite, il faut envisager le recours à la peine capitale.

H. Dumont: Autour de la victime il ne se bâtit pas simplement un mouvement de compassion, il y a également le mouvement politique de la remettre dans le processus judiciaire, c'est-à-dire dans l'exercice des représailles publiques. Il y a sûrement beaucoup de compassion chez ceux et celles qui s'intéressent aujourd'hui aux victimes, ce qui s'explique sans doute par le développement de toute une politique de sensibilisation aux droits humains. Ce qui n'empêche que, d'un autre point de vue, on puisse voir autre chose dans ce processus d'insertion de la victime dans l'exercice du pouvoir de répression — on doit y voir, à mon avis, une stratégie qui contribue à donner des meilleures assises à ce pouvoir. On demande de plus en plus à l'État de punir avec modération. Or, lorsqu'on critique l'exercice de la répression publique, il faut s'attendre que ceux qui en ont le monopole tentent de trouver d'autres justifications, essayant de forger une nouvelle légitimité à l'exercice de ce pouvoir. 